\title{
Okara Supplementation Relieves Fatigue and Muscle Damage that Occur during Exercise Training
}

\author{
Hsiu-Fen Yang', Tim K. Tso ${ }^{1, *}$, Chen-Chih Huang ${ }^{2}$, Wen-Nan Huang ${ }^{3}$ \\ ${ }^{1}$ Department of Food Science, National Chiayi University, Chiayi, Taiwan, R.O.C. \\ ${ }^{2}$ Department of Sports, Health \& Leisure, WuFeng University, Minhsiung, Taiwan, R.O.C. \\ ${ }^{3}$ Department of Allergy, Immunology and Rheumatology, Taichung Veterans General Hospital, Taichung, Taiwan, R.O.C. \\ *Corresponding author: timtso@mail.ncyu.edu.tw
}

Received May 20, 2015; Revised May 31, 2015; Accepted June 04, 2015

\begin{abstract}
Okara, the soybean residue from soy milk production, contains nutrients and functional components. The objective of this study was to investigate the effect of okara supplementation on fatigue and muscle damage in university athletes over an exercise training season. A total of 23 male university baseball players aged 18-23 years attending a summer intensive exercise training program completed an 8-week exercise training-okara intervention study. The supervised exercise training program consisted of physical and technical training including warm-up exercises, physical and skill practice, and pre-game competition practice. Okara supplementation in this study was a randomized, double-blind and crossover dietary intervention in which subjects consumed 2 cookies/day containing $10 \mathrm{~g}$ of soybean or black soybean okara for a period of 6 weeks with a one-week washout period. Blood was drawn 4 times within an 8-week intervention period for measurements of biomarkers for fatigue and muscle damage. The results demonstrated significant differences in blood levels of ammonia, free fatty acids, creatine kinase, myoglobin, and aspartate transferase between baseline and exercise training, indicating that fatigue and muscle damage occurred during exercise training. However, those increased markers of fatigue and muscle damage were significantly decreased after the okara supplementation regardless of whether soybean okara or black soybean okara cookies were consumed. This is the first study to demonstrate that okara supplementation is beneficial to university baseball players who experience exercise training-related fatigue and muscle damage. Fatigue and muscle damage occurring during exercise training were both relieved, indicating that okara cookies are feasible sports nutrition supplements.
\end{abstract}

Keywords: okara, soybean, supplementation, exercise, fatigue, muscle damage

Cite This Article: Hsiu-Fen Yang, Tim K. Tso, Chen-Chih Huang, and Wen-Nan Huang, "Okara Supplementation Relieves Fatigue and Muscle Damage that Occur during Exercise Training." Journal of Food and Nutrition Research, vol. 3, no. 5 (2015): 352-356. doi: 10.12691/jfnr-3-5-10.

\section{Introduction}

Soybean has nutritional value and health functions. Okara, the soybean residue from soy milk production, contains mostly crude fibers, proteins, lipids, and starch or simple carbohydrates [1]. Okara also contains micronutrients and functional components such as isoflavones and phytosterols [2]. However, large quantities of okara produced annually pose a significant disposal problem [1]. Okara in the past was used as animal feed, but recently has been applied to noodles, baked goods, snacks, pickled side dishes, vegetarian additives, and other food products [3]. In addition, previous researches demonstrated that okara could be useful as a functional ingredient with health-promoting attributes $[2,4,5,6,7]$.

When athletes are exercising or training to surpass their previous athletic performance, they often exceed the levels of exertion their bodies can endure, or continuously perform unfamiliar exercises or actions. This can easily result in muscle damage [8], which is often accompanied by delayed onset muscle soreness, reduction of muscle strength and joint range of motion, muscle swelling, and increased concentration of various inflammatory biomarkers [9]. Fortunately, the conditions described above are not life threatening and athletes can return to their normal routine after a period of rest following training [10].

Professional athletes tend to follow the principle of overload during exercise and training. They must train to break the fatigue barrier and enhance physical functions to perform well in intense competitions. However, over the long term, as the intensity of exercise training increases, this may produce negative physical effects harmful to the human body [11]. Fatigue refers to underperformance or any reduction in maximal capacity to perform a task. The sense of fatigue is a subjective feeling and is usually related to tissue injury and a lack of energy [12]. Fatigue or the sense of fatigue occurs after engaging in exercise or various types of stress or challenges. The length of the recovery period can vary according to the type of challenge and the different methods of antioxidant intervention [13]. 
Although a number of previous studies have found that okara has properties beneficial to health, few studies have conducted human trials in relation to okara. To our knowledge, no research has been conducted on the possibility of okara as a functional ingredient with healthpromoting attributes in the field of sports nutrition and medicine. Therefore, the objective of this study was to investigate the effect of okara supplementation on fatigue and muscle damage in university athletes over an exercise training season.

\section{Materials and Methods}

\subsection{Subjects and Research Design}

A total of 23 male university baseball players attending a summer intensive exercise training program were recruited in an 8-week exercise training-okara intervention study. Selection criteria of participants were as follows: no allergic reactions to soybean, non-use of nutritional supplements and nutraceutical products within three months of study entry, no genetic diseases and no family history of chronic diseases, current disease-free status and non-use of general medicine, and willingness to accept the intramural sports summer training modes of exercise training and lifestyle requirements. Written informed consent was obtained from qualified subjects and the university's ethical committee evaluated and approved the study. The supervised exercise training program involved concentric muscle contractions and eccentric muscle contractions, and consisted of physical and technical training including warm-up exercises, physical and skill practice, and pre-game competition practice. A coach and trained health professionals supervised the exercise training program. The training content and methods of coaching were to comply with the arrangements and planning; routine training was conducted in accordance with the team guidelines, and no adjustments were done.

\subsection{Dietary Intervention}

Dietary intervention of this study was designed to determine the effects of okara, provided via cookies, on exercise-induced fatigue and muscle damage in the intensive exercise training program. Soybean okara and black soybean okara were frozen, dried soybean and black soybean residues obtained from a local soybean milk manufacturer. The okara cookies were manufactured according to the general methods of manufacturing cookies sold on the market. Subjects were randomly assigned to the test cookie groups in a crossover design and were asked to consume 2 cookies (labeled A or B)/day, which totaled $10 \mathrm{~g}$ of okara, for a period of 6 weeks. The design included a one-week washout period. During the first treatment period, 12 subjects ate the soybean okara cookies and 11 subjects consumed the black soybean okara cookies. After a one-week washout period, subjects of each group were crossed over to consume different cookies. The cookies were packed in individually labeled bags and provided to the subjects on a 3-day basis. Subjects were asked to return the empty bags or bags containing any uneaten portion of the cookies, and the weight of the cookie was recorded to calculate the amount of cookies consumed per individual during each treatment period. The coach and registered dietitian provided supervision and evaluated whether subjects had any difficulty adhering to the supplementation protocol and any discomfort or side effects. During the dietary intervention, subjects were asked to maintain their habitual diet and lifestyle but to avoid soybean and black soybean products. The subjects were instructed to record their dietary intake over the course of 2 weekdays and 1 weekend day. Subjects discussed their completed food record with a registered dietician to determine accuracy in portion size estimates and details of food intake. Analysis of the dietary record was performed by the registered dietician using the Software System for Nutritional Calculation (EKitchen Business Corporation, Taiwan).

\subsection{Experimental Protocol}

Blood specimens were obtained 4 times within an 8week intervention period at baseline, beginning of exercise training without okara supplementation, the end of the first segment of exercise training with okara supplementation, and the end of the crossover segment of exercise training with okara supplementation, respectively. Biochemical markers for fatigue and muscle damage were measured by the Siemens Dimension RXL Chemistry Analyzer.

\subsection{Statistical Analysis}

The experimental results in this study were statistically analyzed using the Statistical Package for the Social Sciences (SPSS) 10.0 for Windows (SPSS, Inc., Chicago, IL, USA). Tested variables for comparison of means were expressed as mean \pm standard deviation. The paired t test was used to examine the differences among the various parameters before exercise training, after exercise training, and after consumption of the okara cookies. A $p$ value of less than 0.05 indicated a statistically significant difference.

\section{Results}

The compositions of soybean okara and black soybean okara are shown in Table 1. Previous studies did not differentiate between black soybean okara and soybean okara. In this study, black soybean okara had a higher amount of protein compared to soybean okara, whereas the fiber content of black soybean okara was lower than that of soybean okara. The daily energy provided by these cookies was $360 \mathrm{kcal}$, and the amount of dietary fiber was approximately $5.2 \mathrm{~g}$ for the soybean okara cookies and 3.5 $\mathrm{g}$ for the black soybean okara cookies. The okara cookies consumed by the subjects in the study were comparable to regular cookies sold on the market in terms of ingredients and manufacturing methods. These cookies were also tasted and judged by members of the general public in the same age range as the subjects. The cookie recipe that received the best reviews was used to make the okara cookies used in the experiment.

A total of 23 male university baseball players aged 1823 years attending a summer intensive exercise training program completed an 8-week exercise training-okara intervention study. Participating athletes included six pitchers, five infielders, seven outfielders, two shortstops, 
and three catchers. The anthropometric and biochemical measurements of participating athletes are shown in Table 2. Serum total cholesterol, triglyceride, low density lipoprotein-cholesterol and r-glutamyl transferase were significantly decreased during the exercise training. The biochemical markers of fatigue and muscle damage at baseline and during exercise training with and without okara supplementation are shown in Table 3 . The results demonstrated significant differences in blood levels of ammonia, free fatty acids, creatine kinase, myoglobin, and aspartate transferase between baseline and exercise training. After the one-week training, creatine kinase, myoglobin, ammonia, aspartate transferase, and free fatty acids significantly increased, indicating that fatigue and muscle damage occurred during exercise training. However, those increased markers of fatigue and muscle damage were significantly decreased after the okara supplementation. This indicates that okara, either soybean okara or black soybean okara, can reduce muscle damage and delay fatigue during exercise training.

Table 1. The compositions of soybean okara and black soybean okara used in the study

\begin{tabular}{|c|c|c|}
\hline & Soybean okara & Black soybean okara \\
\hline \multicolumn{3}{|c|}{ Proximate composition g/100g, dry weight } \\
\hline Protein & 19.86 & 25.81 \\
\hline Crude fat/oil & 10.48 & 11.08 \\
\hline Carbohydrates & 63.17 & 55.86 \\
\hline Crude fiber & 13.75 & 8.59 \\
\hline \multicolumn{3}{|c|}{ Amino acid composition mg/g, dry weight; (\%) } \\
\hline Glutamate & $37.01(17.6)$ & $50.31(18.0)$ \\
\hline Aspartate & $23.86(11.4)$ & $35.57(12.7)$ \\
\hline Leucine & $17.32(8.2)$ & $22.38(8.0)$ \\
\hline Lysine & $14.88(7.1)$ & $19.09(6.8)$ \\
\hline Arginine & $14.13(6.7)$ & 21.96 (7.9) \\
\hline Serine & $12.35(5.9)$ & $15.67(5.6)$ \\
\hline Valine & $11.62(5.5)$ & $14.78(5.3)$ \\
\hline Phenylalanine & $11.42(5.4)$ & $15(5.4)$ \\
\hline Glycine & $10.83(5.2)$ & $13.45(4.8)$ \\
\hline Proline & $10.78(5.1)$ & $14.16(5.1)$ \\
\hline Alanine & $10.21(4.9)$ & 13.07 (4.7) \\
\hline Threonine & $10.03(4.8)$ & $12.4(4.4)$ \\
\hline Isoleucine & $9.06(4.3)$ & $12.24(4.38)$ \\
\hline Histidine & $6.69(3.2)$ & $8.34(3.0)$ \\
\hline Tyrosine & 6.16 (2.9) & 8.13 (2.9) \\
\hline Cysteine & $2.60(1.2)$ & 3.41 (1.2) \\
\hline Methionine & $1.25(0.6)$ & $2.06(0.7)$ \\
\hline Total & $210.2(100.0)$ & $279(100.0)$ \\
\hline
\end{tabular}

Table 2. The anthropometric and biochemical measurements of participating athletes $(n=23)^{a}$

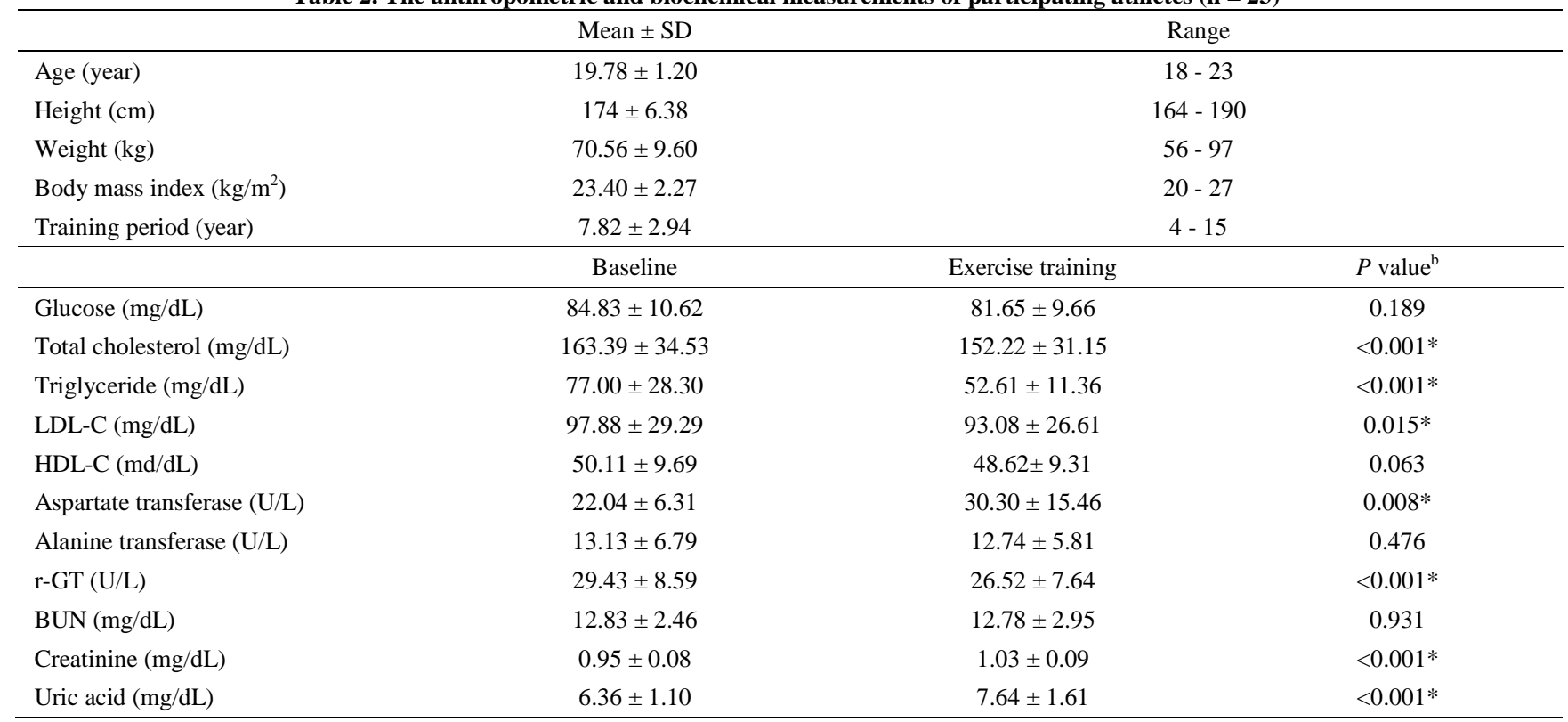

LDL-C, Low density lipoprotein-cholesterol; HDL-C, High density lipoprotein-cholesterol; r-GT, Gamma-glutamyl transferase;

BUN, Blood urea nitrogen

${ }^{\mathrm{a}}$ Values are expressed as mean \pm standard deviation.

${ }^{\mathrm{b}}$ Statistical significance of mean (baseline vs. exercise training no supplementation, ${ }^{*} P<0.05$ ) was determined by the paired $\mathrm{t}$ test. 
Table 3. The biochemical markers of fatigue and muscle damage at baseline and during exercise training with and without okara supplementation $(\mathbf{n}=23)^{\mathrm{a}}$

\begin{tabular}{|c|c|c|c|c|}
\hline & \multirow[t]{2}{*}{ Baseline } & \multirow[t]{2}{*}{$\begin{array}{l}\text { Exercise training } \\
\text { no supplementation }\end{array}$} & \multicolumn{2}{|c|}{$\begin{array}{l}\text { Exercise training } \\
\text { supplementation }\end{array}$} \\
\hline & & & soybean okara & black soybean okara \\
\hline Glucose (mg/dL) & $84.83 \pm 10.62$ & $81.65 \pm 9.66$ & $86.70 \pm 6.06^{*}$ & $87.57 \pm 5.99^{*}$ \\
\hline Lactic acid (mmol/L) & $2.41 \pm 0.45$ & $1.62 \pm 0.65$ & $1.48 \pm 0.41$ & $1.75 \pm 0.55$ \\
\hline Ammonia $(\mu \mathrm{g} / \mathrm{dL})$ & $122.57 \pm 29.54$ & $166.30 \pm 37.35^{\#}$ & $141.35 \pm 26.53^{*}$ & $131.48 \pm 22.22^{*}$ \\
\hline Free fatty acid (mmol/L) & $0.39 \pm 0.14$ & $0.71 \pm 0.31^{\#}$ & $0.43 \pm 0.16^{*}$ & $0.44 \pm 0.12^{*}$ \\
\hline Creatine kinase (U/L) & $111.00 \pm 49.44$ & $447.30 \pm 396.75^{\#}$ & $174.65 \pm 79.50^{*}$ & $186.65 \pm 101.97^{*}$ \\
\hline Lactate dehydrogenase (U/L) & $146.89 \pm 17.55$ & $151.68 \pm 27.97$ & $140.37 \pm 22.96$ & $146.26 \pm 28.57$ \\
\hline Myoglobin (ng/mL) & $23.71 \pm 7.13$ & $61.17 \pm 18.03^{\#}$ & $22.38 \pm 3.97^{*}$ & $22.62 \pm 5.03^{*}$ \\
\hline Aspartate transferase (U/L) & $22.04 \pm 6.31$ & $30.30 \pm 15.46^{\#}$ & $22.70 \pm 6.46^{*}$ & $21.87 \pm 5.26^{*}$ \\
\hline
\end{tabular}

${ }^{\mathrm{a}}$ Values are expressed as mean \pm standard deviation.

\#Statistical significance of mean (baseline vs. exercise training no supplementation, $P<0.05$ ) was determined by the paired t test.

*Statistical significance of mean (exercise training no supplementation vs. exercise training with either soybean okara supplementation or black soybean okara supplementation, $P<0.05$ ) was determined by the paired t test.

\section{Discussion}

To our knowledge, this is the first study to demonstrate that okara supplementation is beneficial to university baseball players who experience exercise training-related fatigue and muscle damage. The major finding was that the elevation of blood levels of biochemical markers for fatigue and muscle damage including ammonia, free fatty acids, creatine kinase, myoglobin, and aspartate transferase during exercise training significantly decreased after okara supplementation.

Ammonia in the blood is an important indicator of exercise fatigue [14]. Vigorous exercise generates a large amount of ammonia in the blood, and the increased concentration of ammonia increases the occurrence of fatigue [14]. The research period in this study was the summer training period for university baseball players. A majority of the training involved resistance-type exercise; therefore, the conditions of fatigue and muscle damage were more easily observed. The results of this study showed that after the consumption of okara cookies during training, ammonia concentration was significantly decreased. This indicated that okara intake aids in significantly reducing exercise fatigue.

The concentration of creatine kinase and myoglobin in plasma is often used as an indicator of muscle damage [15, 16]. Resistance training that involves more eccentric muscle contractions can result in muscle damage, increasing the concentration of these proteins in the blood. Endurance training is less likely to result in this type of damage [16]. This study found that okara supplements significantly decreased the concentration of creatine kinase and myoglobin during training, indicating that these supplements served to defer muscle damage.

Free fatty acids in the blood are a key source of energy for low/moderate intensity exercise [17]. During exercise, the concentration of free fatty acids in the blood increases. Free fatty acids and tryptophan compete for binding sites on albumin, increasing the concentration of free tryptophan in the blood and thereby increasing the concentration of serotonin in the brain [18]. The results of this study showed that exercise increases the concentration of free fatty acids in the blood, but consumption of soybean or black soybean okara supplements during exercise reduced the concentration of free fatty acids to pre-exercise levels. This showed that okara supplements aid significantly in relieving exercise-induced fatigue. However, this study did not research the mechanism through which this occurs. Therefore, the cause-effect relationship among okara supplements, free fatty acids, and fatigue is yet to be clarified.

Lactic acid is mainly generated during acute anaerobic exercise. When exercise is overly demanding and oxygen supply is inadequate, the body uses an anaerobic metabolic mechanism to generate energy [19]. In this experiment, lactic dehydrogenase did not significantly change at any stage, whether before training, after training, or during the consumption of okara cookies while training. This may be because this study focused on researching the long-term influence of exercise training; therefore, no significant differences were detected in the metabolic markers of acute supply.

As indicated by previous studies, in recent years, researchers have focused on the influence of branchedchain amino acids (BCAAs) on muscle protein and the immune system. Research has shown that using BCAA supplements before and after exercise aids in reducing muscle damage and promoting muscle protein synthesis [20]. Other studies have shown that arginine improves vascular endothelial proliferation. Initial evidence has also demonstrated that arginine may change the metabolic action of skeletal muscle by increasing nitric oxide. Increasing arginine during exercise can enhance capillary growth in muscles [21]. Analysis of both soybean and black soybean okara used in this study showed that the okara contained 14.13 - $21.96 \mathrm{mg} / \mathrm{g}$ of arginine, 17.32 $22.38 \mathrm{mg} / \mathrm{g}$ of leucine, 11.62 - $14.78 \mathrm{mg} / \mathrm{g}$ of valine, and 9.06 - $12.24 \mathrm{mg} / \mathrm{g}$ of isoleucine (Table 1). Past studies have established that supplements of arginine and BCAAs are significantly beneficial to muscle recovery and assist in mitigating fatigue and muscle damage during exercise training. Consequently, we speculated that arginine and BCAAs in the okara used in this study eased fatigue and muscle damage during exercise training.

\section{Conclusions}

During the eight-week exercise training period in this study, creatine kinase, myoglobin, ammonia, aspartate transferase, and free fatty acids were significantly reduced in all the athletes after okara supplementation. Fatigue and muscle damage occurring during exercise training were both relieved, indicating that okara cookies are feasible 
sports nutrition supplements. However, the relevant mechanisms of okara that mitigate fatigue and muscle damage in exercise training require further research.

\section{Acknowledgement}

This study was supported by a research grant from the Ministry of Science and Technology of Taiwan.

\section{Statement of Competing Interests}

The authors have no competing interests.

\section{References}

[1] O'Toole, D.K, "Characteristics and use of okara, the soybean residue from soy milk production--a review," J Agric Food Chem, 47. 363-371. 1999.

[2] Jiménez-Escrig, A., Tenorio, M.D., Espinosa-Martos, I., Rupérez, $P$, "Health-promoting effects of a dietary fiber concentrate from the soybean byproduct okara in rats," J Agric Food Chem, 56. 7495-7501. 2008.

[3] Katayama, M., Wilson, L.A, "Utilization of okara, a byproduct from soymilk production, through the development of soy-based snack food," J Food Sci, 73. S152-S157. 2008.

[4] Matsuo, M, "Chemical components, palatability, antioxidant activity and antimutagenicity of oncom miso using a mixture of fermented soybeans and okara with Neurospora intermedia," $J$ Nutr Sci Vitaminol (Tokyo), 52. 216-222. 2006.

[5] Matsuo, M, "In vivo antioxidant activity of Okara Koji, a fermented okara, by Aspergillus oryzae," Biosci Biotechnol Biochem, 61. 1968-1972. 1997.

[6] Yokota, T., Ohami, H., Ohishi, H., Hattori, T., Watanabe, K, "Repression of acute gastric mucosal lesions by antioxidantcontaining fraction from fermented products of okara (bean-curd residue)," J Nutr Sci Vitaminol (Tokyo), 42. 167-172. 1996.
[7] Matsumoto, K., Watanabe, Y., Yokoyama, S, "Okara, soybean residue, prevents obesity in a diet-induced murine obesity model," Biosci Biotechnol Biochem, 71. 720-727. 2007.

[8] Nosaka, K., Newton, M, "Concentric or eccentric training effect on eccentric exercise-induced muscle damage," Med Sci Sports Exerc, 34. 63-69. 2002.

[9] Pedersen, B.K., Ostrowski, K., Rohde, T., Bruunsgaard, H, "The cytokine response to strenuous exercise," Can J Physiol Pharmacol, 76. 505-511. 1998.

[10] Falvo, M.J., Schilling, B.K., Bloomer, R.J., Smith, W.A., Creasy, A.C, "Efficacy of prior eccentric exercise in attenuating impaired exercise performance after muscle injury in resistance trained men," J Strength Cond Res, 21. 1053-1060. 2007.

[11] Evans, W.J., Lambert, C.P, "Physiological basis of fatigue," Am J Phys Med Rehabil, 86(Suppl. 1). S29-S46. 2007.

[12] Vøllestad, N.K, "Measurement of human muscle fatigue," $J$ Neurosci Methods, 74. 219-227. 1997.

[13] Strobel, N.A., Peake, J.M., Matsumoto, A., Marsh, S.A., Coombes, J.S., Wadley, G.D, "Antioxidant supplementation reduces skeletal muscle mitochondrial biogenesis," Med Sci Sports Exerc, 43.1017-1024. 2011.

[14] Banister, E.W., Cameron, B.J, "Exercise-induced hyperammonemia: peripheral and central effects," Int J Sports Med, 11(Suppl. 2). 129-142. 1990.

[15] Brancaccio, P., Lippi, G., Maffulli, N, "Biochemical markers of muscular damage,” Clin Chem Lab Med, 48. 757-767. 2010.

[16] Brancaccio, P., Maffulli, N., Limongelli, F.M, "Creatine kinase monitoring in sport medicine," Br Med Bull, 81-82. 209-230. 2007.

[17] Wigernaes, I., Hostmark, A.T., Stromme, S.B., Kierulf, P., Birkeland, K, "Active recovery and post-exercise white blood cell count, free fatty acids, and hormones in endurance athletes," Eur $J$ Appl Physiol, 84. 358-366. 2001.

[18] Chaouloff, F, "Effects of acute physical exercise on central serotonergic systems," Med Sci Sports Exerc, 29. 58-62. 1997.

[19] Sola-Penna, M, “Metabolic regulation by lactate," IUBMB Life, 60. 605-608. 2008.

[20] Negro, M., Giardina, S., Marzani, B., Marzatico, F, "Branchedchain amino acid supplementation does not enhance athletic performance but affects muscle recovery and the immune system," J Sports Med Phys Fitness, 48. 347-351. 2008.

[21] McConell, G.K, "Effects of L-arginine supplementation on exercise metabolism," Curr Opin Clin Nutr Metab Care, 10. 46-51. 2007. 